\title{
ALDH1A1 overexpression is associated with the progression and prognosis in gastric cancer
}

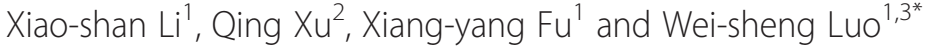

\begin{abstract}
Background: Aldehyde dehydrogenase 1 family member A1 (ALDH1A1) is a cancer stem cell marker, and its expression correlates with prognosis in a number of malignancies. The aim of this study is to determine the relationship of ALDH1A1 expression with clinicopathological parameters and prognosis in gastric cancer.

Methods: ALDH1A1 and matrix metallopeptidase 9 (MMP-9) was evaluated by immunohistochemistry in 216 gastric carcinoma samples. The association between expression of ALDH1A1 and MMP-9, clinicopathological parameters, and prognosis of gastric cancer was examined.
\end{abstract}

Results: ALDH1A1 protein expression was significantly associated with depth invasion, lymph node metastasis and stage of disease (all $P<0.05$ ). Both univariate and multivariate analyses revealed that ALDH1A1 was an independent prognostic factor for both overall survival (OS) and recurrence-free survival (RFS) (both $P<0.001$ ). Furthermore, ALDH1A1 overexpression was associated with poor prognosis in patients subgroups stratified by tumor size, depth invasion and lymph node metastasis. Moreover, ALDH1A1 was significantly correlated with MMP-9 among 216 gastric cancer tissues $(P<0.001)$. Patients who had ALDH1A1 overexpression, in which tumor cells displayed high invasiveness, had poor OS and shorter RFS.

Conclusion: ALDH1A1 plays an important role in tumor aggressiveness and prognosis, and may act as a promising target for prognostic prediction.

\section{Background}

Gastric cancer is one of the leading causes of cancerrelated death worldwide due to its frequency, poor prognosis and limited treatment options [1]. Complete resection of the tumor and adjacent lymph nodes is the only effective curative treatment [2]. Unfortunately, after a complete resection, the 5-year survival rate remains low [3]. Several studies have shown that various genetic and epigenetic alterations are involved in the course of carcinogenesis and progression of gastric cancer [4-6]. However, the molecular mechanism involved in the development of gastric cancer remains unclear.

Aldehyde dehydrogenases (ALDHs) are a group of proteins that share highly conserved sequences essential for function. Each subunit contains a catalytic domain, a

\footnotetext{
* Correspondence: wsluo58@126.com

'Department of Gastroenterology, Affiliated Hospital of Guilin Medical University, Guilin 541004, Guangxi Zhuang Autonomous Region, China ${ }^{3}$ Department of Spleen and stomach diseases, The First Affiliated Hospital of Guangxi University of Chinese Medicine, Nanning 530200, Guangxi Zhuang Autonomous Region, China

Full list of author information is available at the end of the article
}

cofactor binding domain, and a bridging domain. Each subtype's catalytic pocket has a specificity for a particular substrate [7]. The human ALDH superfamily currently consists of 19 known putatively functional genes in 11 families and 4 subfamilies with distinct chromosomal locations [7-9]. The ALDH enzymes can be found in the cytosol, nucleus, mitochrondria, and endoplasmic reticulum. The function of ALDH is to modulate several cell functions, including proliferation, differentiation, and survival, as well as the cellular response to oxidative stress. It has been reported that the ALDH enzymes that are involved in normal stem cells as well as cancer stem cells include the ALDH1 family, ALDH2*2, ALDH3A1, ALDH4A1 and ALDH7A1 [7]. In particular, ALDH1 has been used as a marker to identify and isolate normal and cancer stem cells. It has been known that the ALDH1 subfamily comprises of ALDH1A1, ALDH1A2 and ALDH1A3.

ALDH1A1 is a cytosolic enzyme responsible for oxidizing a variety of intracellular aldehydes to carboxylic acids [10]. It also plays an important role in the detoxification of peroxidic aldehydes produced by ultraviolet 
light absorption, protecting the lens of the eye. In addition, it exhibits high activity for oxidation of aldophosphamide and has a role in the detoxification of some commonly used anticancer drugs [11]. Recently, it has been reported that ALDH1A1 has been related to adverse prognosis in several human malignancies, including breast cancer, lung cancer, ovarian cancer and esophageal cancer [12-15]. However, the role of ALDH1A1 on the prognosis of patients with gastric cancer remains unclear.

In the present study, we assessed the expression of ALDH1A1 in gastric cancer tissues by immunohistochemistry. Correlation of ALDH1A1 with clinicopathological parameters and survival of gastric cancer patients were then analyzed. In addition, it has been reported that MMP-9 plays an important role in gastric cancer recurrence and prognosis [16]. Therefore, we also investigated the relationship of ALDH1A1 and MMP-9 protein in gastric cancer.

\section{Methods}

\section{Patients and specimens}

The study was approved by the Institutional Review Board and Human Ethics Committee of Affiliated Hospital of Guilin Medical University. Written consent for using the samples for research purposes was obtained from all patients prior to surgery.

Gastric carcinoma tissues were obtained from gastrectomy specimens of 216 patients from the department of general surgery, the Affiliated Hospital of Guilin Medical University (Guilin, China). All the operations were performed between January 2005 and December 2008. The eligibility criteria of the current study were as follows: (1) a pathologic examination confirming the presence of gastric cancer and experienced radical surgery, (2) complete basic clinical data, (3) the absence of any prior treatment for cancer, and (4) no serious complications or other malignant disease. There were 140 males and 76 females (mean age, 57.0 years; range, 22-82 years). Relevant clinical pathologic features (Table 1) were all obtained from the patients' files. Tumor stage was classified according to the 7th Union International Cancer Control (UICC) TNM staging system [17].

\section{Immunohistochemistry staining}

A total of 216 gastric carcinoma samples were used in the immunohistochemistry (IHC) analysis. According to protocol [18] for IHC on paraffin-embedded tissue sections, paraffin-embedded blocks were sectioned at about $4 \mu \mathrm{m}$ thickness. Slides were baked at $60^{\circ} \mathrm{C}$ for $2 \mathrm{~h}$, deparaffinized with xylene and rehydrated using an alcohol gradient (100\% alcohol, 95\% alcohol, $80 \%$ alcohol, and $70 \%$ alcohol). The tissue slides were then treated with $3 \%$ hydrogen peroxide in methanol for 30 min to quench endogenous peroxidase activity, and the antigens were
Table 1 Clinicopathologic correlation of ALDH1A1 expression in $\mathbf{2 1 6}$ gastric cancer

\begin{tabular}{|c|c|c|c|c|}
\hline \multirow[t]{2}{*}{ Characteristics } & \multirow{2}{*}{$\begin{array}{c}\text { No. of } \\
\text { patients }\end{array}$} & \multicolumn{2}{|c|}{ ALDH1A1 expression (\%) } & \multirow[t]{2}{*}{$P$-value } \\
\hline & & Negative & Positive & \\
\hline \multicolumn{5}{|l|}{ Gender } \\
\hline Male & 140 & $64(45.7 \%)$ & $76(54.3 \%)$ & \\
\hline Female & 76 & $44(57.9 \%)$ & $32(42.1 \%)$ & 0.087 \\
\hline \multicolumn{5}{|l|}{ Age (years) } \\
\hline$\leq 60$ & 136 & $74(54.4 \%)$ & $62(45.6 \%)$ & \\
\hline$>60$ & 80 & $34(42.5 \%)$ & $46(57.5 \%)$ & 0.091 \\
\hline \multicolumn{5}{|l|}{ Size $(\mathrm{cm})$} \\
\hline$\leq 5.0$ & 139 & $76(54.7 \%)$ & $63(45.3 \%)$ & \\
\hline$>5.0$ & 77 & $32(41.6 \%)$ & $45(58.4 \%)$ & 0.065 \\
\hline \multicolumn{5}{|l|}{ Tumor site } \\
\hline Upper & 86 & $36(41.9 \%)$ & $50(58.1 \%)$ & \\
\hline Middle/Lower & 130 & $72(55.4 \%)$ & $58(44.6 \%)$ & 0.052 \\
\hline \multicolumn{5}{|l|}{ Differentiation } \\
\hline Well/Moderate & 96 & $41(42.7 \%)$ & $55(57.3 \%)$ & \\
\hline Poor & 120 & $67(55.8 \%)$ & $53(44.2 \%)$ & 0.055 \\
\hline \multicolumn{5}{|l|}{ Depth of invasion } \\
\hline $\mathrm{T} 1 / \mathrm{T} 2$ & 81 & $58(71.6 \%)$ & $23(28.4 \%)$ & \\
\hline $\mathrm{T} 3 / \mathrm{T} 4$ & 135 & $50(37.0 \%)$ & $85(63.0 \%)$ & $<0.001$ \\
\hline \multicolumn{5}{|c|}{ Lymph node metastasis } \\
\hline Negative & 59 & $46(78.0 \%)$ & $13(22.0 \%)$ & \\
\hline Positive & 157 & $62(39.5 \%)$ & $95(60.5 \%)$ & $<0.001$ \\
\hline \multicolumn{5}{|l|}{ Stages } \\
\hline$|/| \mid$ & 71 & $52(73.2 \%)$ & $19(26.8 \%)$ & \\
\hline III & 145 & $56(38.6 \%)$ & 89 (61.4\%) & $<0.001$ \\
\hline
\end{tabular}

retrieved in $0.01 \mathrm{M}$ sodium citrate buffer ( $\mathrm{pH}$ 6.0) using a microwave oven. After $30 \mathrm{~min}$ of preincubation in 10\% normal goat serum to prevent nonspecific staining, the samples were incubated overnight using a primary antibody, either anti-ALDH1A1 (Abcam, \#ab52492, UK, dilution 1:200) or anti-MMP-9 (Abcam, \#ab38898, UK, dilution 1:200), in a humidified container at $4^{\circ} \mathrm{C}$. The tissue slides were treated with a non-biotin horseradishperoxidase detection system according to the manufacturer's instructions (Gene Tech). The IHC results were evaluated by two independent investigators blinded to the patients' identity and clinical status. In discrepant cases, a pathologist reviewed the cases, and a consensus was reached.

ALDH1A1 and MMP-9 staining intensities were rated on a scale of $0-3$ according to the percentage of positive tumor $(0,<5 \%$ positive cells; $1,5-10 \% ; 2,11-50 \%$; or $3,>$ $50 \%)$. The expression is very low for 0 , low for 1 , moderate for 2 and high for 3 (Figure 1). ALDH1A1 and MMP-9 


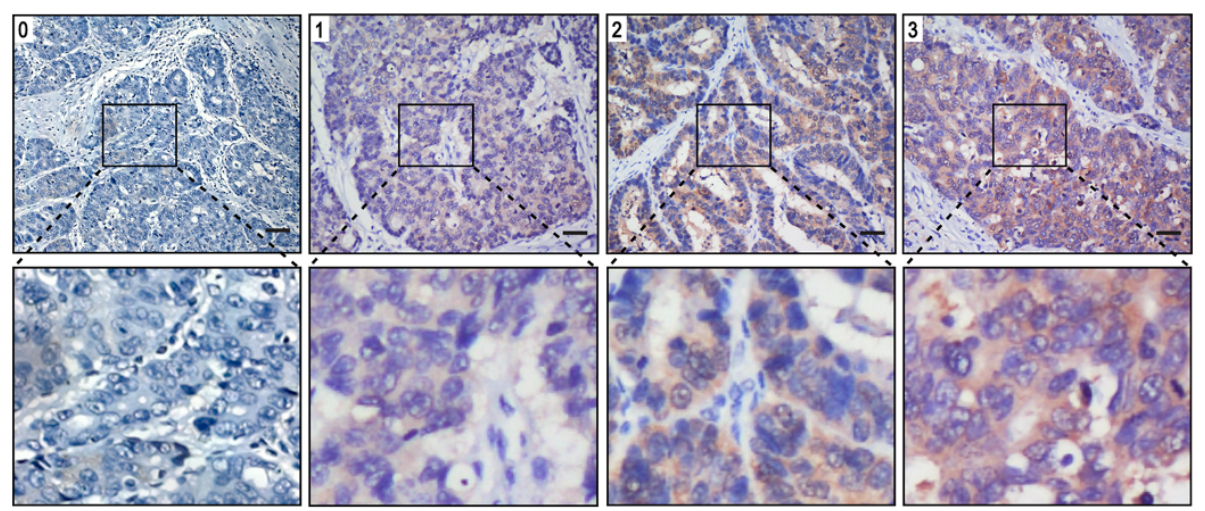

Figure 1 Gastric cancer tissue illustrating the range of intensities of ALDH1A1 immunostaining from 0 to 3. The lower panels represent magnified pictures of boxed area in the corresponding upper panels. The scale bar represents $50 \mu \mathrm{m}$.

expression were classified as negative for scores $\leq 1$ and positive for scores $\geq 2$.

\section{Follow-up}

The follow-up duration was defined as the interval between the date of operation and the date of death or last follow-up. The study was censored on 30 September 2013. The median follow-up period was 27.0 months (range, 4-82 months) in 216 patients. All the patients were followed up every 1-3 months in the first year and every 3-6 months thereafter. Recurrence were confirmed by tumor markers levels including CEA, AFP, CA199, CA125 and CA724, B-type ultrasonic inspection every 3 moths, and computed tomography $(\mathrm{CT})$ or magnetic resonance imaging (MRI) every 6 months after gastrectomy. The main causes of death were gastric cancer recurrence. Overall survival (OS) was calculated from the date of surgery to the date of death or last follow-up. Recurrencefree survival (RFS) was defined as from the date of surgery until the date of relapse or from the period of resection to the date of the last observation taken.

\section{Statistical analysis}

All statistical analyses were performed using the SPSS software (version 16.0; Chicago, IL, USA). Interdependence between ALDH1A1 status and clinical data was calculated using the chi-square test, and displayed in cross-tables. Correlation of ALDH1A1 with MMP-9 was calculated by Pearson $X^{2}$ test. Survival curves were plotted using the Kaplan-Meier method and analyzed using the log-rank test. All reported $P$ values were two-sided and $P<0.05$ was considered statistically significant.

\section{Results}

\section{The association of ALDH1A1 with clinicopathological} variables

To elucidate the biological significance of ALDH1A1 in gastric cancer, we examined the immunohistochemical expression of ALDH1A1 in gastric cancer tissues (Figure 1). ALDH1A1 staining mainly located in cytoplasm of tumor cells. The positive rate of ALDH1A1 was 50.0\% (108/216) in gastric cancer samples.

According to the results of immunohistochemistry, we correlated ALDH1A1 status in 216 gastric cancer specimens with clinicopathologic parameters (Table 1). Our analyses showed that the level of ALDH1A1 in gastric cancer was significantly correlated with depth of invasion $(P<0.001)$, lymph node metastasis $(P<0.001)$, and stage of disease $(P<0.001)$, but was not associated with gender, age, tumor size, tumor site and grade of differentiation $(P>0.05)$ (Table 1$)$. Notably, the correlation of ALDH1A1 with prominent serosal invasion and lymph node metastasis positivity suggested a potential role of ALDH1A1 in increased invasion and metastasis of gastric cancer.

\section{Effect of tumor ALDH1A1 protein level on prognosis}

To further determine the effect of ALDH1A1 overexpression on the OS and RFS, we first performed univariate analysis of traditional clinicopathologic variables for prognosis. The results of the univariate analysis are shown in Table 2. Overexpression of ALDH1A1 $(P<0.001)$, larger tumor size $(P<0.001)$, prominent serosal invasion $(P<0.001)$ and lymph node metastasis $(P<0.001)$ were significantly associated with the poor OS rate of gastric cancer patients. In addition, Kaplan-Meier analysis demonstrated that ALDH1A1 overexpression $(P<0.001)$, larger tumor size $(P=0.001)$, tumor site $(P=0.047)$, prominent serosal invasion $(P<0.001)$ and lymph node metastasis $(P<0.001)$ were negative prognostic factors for RFS in gastric cancer patients (Table 2). Furthermore, to evaluate the independent impact of ALDH1A1 overexpression on OS and RFS, a multivariate Cox regression model adjusted for tumor size, tumor site, depth of invasion, lymph node metastasis and ALDH1A1 expression was performed. Our results showed that ALDH1A1 
Table 2 Predictive variables for overall survival and recurrence-free survival of $\mathbf{2 1 6}$ patients with gastric cancer

\begin{tabular}{|c|c|c|c|c|c|c|c|}
\hline \multirow[t]{2}{*}{ Variables } & \multirow[t]{2}{*}{ No. of patients } & \multicolumn{2}{|c|}{ OS rate (\%) } & \multirow[t]{2}{*}{$P$-value } & \multicolumn{2}{|c|}{ RFS rate (\%) } & \multirow[t]{2}{*}{$P$-value } \\
\hline & & $3 y$ & $5 y$ & & $3 y$ & $5 y$ & \\
\hline \multicolumn{8}{|l|}{ Gender } \\
\hline Male & 140 & 46.9 & 39.6 & & 41.5 & 35.4 & \\
\hline Female & 76 & 54.0 & 43.1 & 0.308 & 44.4 & 41.2 & 0.381 \\
\hline \multicolumn{8}{|l|}{ Age (years) } \\
\hline$\leq 60$ & 136 & 49.8 & 43.9 & & 45.3 & 42.2 & \\
\hline$>60$ & 80 & 48.9 & 36.1 & 0.338 & 38.1 & 30.6 & 0.391 \\
\hline \multicolumn{8}{|l|}{ Size $(\mathrm{cm})$} \\
\hline$\leq 5.0$ & 139 & 55.6 & 47.4 & & 50.4 & 45.5 & \\
\hline$>5.0$ & 77 & 38.1 & 28.8 & 0.001 & 28.3 & 23.3 & $<0.001$ \\
\hline \multicolumn{8}{|l|}{ Tumor site } \\
\hline Upper & 86 & 45.9 & 33.8 & & 34.6 & 28.6 & \\
\hline Middle/Lower & 130 & 51.8 & 45.7 & 0.074 & 47.8 & 43.7 & 0.047 \\
\hline \multicolumn{8}{|l|}{ Differentiation } \\
\hline Well/Moderate & 96 & 47.8 & 39.7 & & 41.2 & 34.6 & \\
\hline Poor & 120 & 50.7 & 41.6 & 0.673 & 43.6 & 40.1 & 0.822 \\
\hline \multicolumn{8}{|l|}{ Depth of invasion } \\
\hline $\mathrm{T} 1 / \mathrm{T} 2$ & 81 & 71.4 & 66.5 & & 70.2 & 65.3 & \\
\hline $\mathrm{T} 3 / \mathrm{T} 4$ & 135 & 36.2 & 25.5 & $<0.001$ & 25.6 & 20.8 & $<0.001$ \\
\hline \multicolumn{8}{|c|}{ Lymph node metastasis } \\
\hline Negative & 59 & 83.5 & 79.2 & & 82.0 & 77.5 & \\
\hline Positive & 157 & 36.5 & 26.6 & $<0.001$ & 27.5 & 22.6 & $<0.001$ \\
\hline \multicolumn{8}{|c|}{ ALDH1A1 protein expression } \\
\hline Negative & 108 & 69.3 & 57.1 & & 60.5 & 54.8 & \\
\hline Positive & 108 & 28.7 & 23.9 & $<0.001$ & 23.7 & 19.2 & $<0.001$ \\
\hline
\end{tabular}

expression was a poor independent prognostic factor for OS in gastric cancer patients (hazard ratio, 2.037; 95\% CI, 1.407 - 2.950). In addition, positive ALDH1A1 expression patients were almost 2.0 times more likely to suffer from relapse than those with negative ALDH1A1 expression (hazard ratio, 1.945; 95\% CI, 1.346 - 2.812). Tumor size, depth of invasion and lymph node metastasis all had independent prognostic value in the multivariate analysis (Table 3).

Survival analysis showed that OS and RFS were significant different among 216 patients according to the expression of ALDH1A1 $(P<0.001, P<0.001)$ (Figure $2 \mathrm{~A})$. The postoperative median OS and RFS were 27.0 months and 19.0 months, respectively. The postoperative median OS times in ALDH1A1-positive $(\mathrm{n}=108)$ and ALDH1A1negative $(n=108)$ gastric cancer patients subgroup were 12.0 months and 42.0 months, and the median of the RFS times were 9.0 months and 39.0 months. In addition, the OS and RFS rates at 5 years were $23.9 \%$ and $19.2 \%$ for ALDH1A1-positive patients compared with $57.1 \%$ and
54.8\% for ALDH1A1-negative patients, respectively (both $P<0.001$; Table 2).

To further evaluate the prognostic value of ALDH1A1 in different subgroups, patients were stratified according to tumor size (Figure 2B,C), depth of invasion (Figure 2D,E) and lymph node metastasis (Figure 2F,G). The expression of ALDH1A1 maintained its prognostic value in predicting shorter OS and RFS in all of these subgroups for except OS in T1/T2 subgroup $(P=0.054)$. Therefore, it appears that ALDH1A1 may serve as a powerful prognostic factor for patients with gastric cancer in different risk groups.

\section{ALDH1A1 overexpression predict poor prognosis independent of tumor invasiveness}

To better understand the clinical significance of ALDH1A1 on aggressiveness in gastric cancer, we investigated the relationship of ALDH1A1 and MMP-9 protein expression in gastric cancer. 
Table 3 Cox multivariate analysis of contributory factors to prognosis among 216 gastric cancer patients after gastrectomy

\begin{tabular}{|c|c|c|c|c|}
\hline Variables & $\beta$ & SE & Hazard ratio $(95 \% \mathrm{Cl})$ & $P$-value ${ }^{a}$ \\
\hline \multicolumn{5}{|l|}{ OS } \\
\hline Tumor size & 0.410 & 0.177 & $1.507(1.065 \sim 2.133)$ & 0.021 \\
\hline Depth of invasion & 0.576 & 0.255 & $1.779(1.079 \sim 2.931)$ & 0.024 \\
\hline Lymph node metastasis & 1.018 & 0.341 & $2.767(1.417 \sim 5.403)$ & 0.003 \\
\hline ALDH1A1 protein expression & 0.712 & 0.189 & $2.037(1.407 \sim 2.950)$ & $<0.001$ \\
\hline \multicolumn{5}{|l|}{ RFS } \\
\hline Tumor size & 0.411 & 0.178 & $1.508(1.064 \sim 2.138)$ & 0.021 \\
\hline Tumor site & 0.043 & 0.180 & $1.044(0.733 \sim 1.486)$ & 0.813 \\
\hline Depth of invasion & 0.649 & 0.256 & $1.913(1.158 \sim 3.162)$ & 0.011 \\
\hline Lymph node metastasis & 1.063 & 0.338 & $2.894(1.491 \sim 5.619)$ & 0.002 \\
\hline ALDH1A1 protein expression & 0.665 & 0.188 & $1.945(1.346 \sim 2.812)$ & $<0.001$ \\
\hline
\end{tabular}

Abbreviations: $A L D H 1 A 1$ aldehyde dehydrogenase 1 family member $\mathrm{A} 1, \mathrm{Cl}$ confidence interval.

${ }^{a}$ Cox proportional hazards regression model.

The positive rates of ALDH1A1 were $63.0 \%$ and $60.5 \%$ in the more prominent serosal invasion group (T3/T4) and more frequent lymph node involvement group (N1-3), while there were only $28.4 \%$ and $22.0 \%$ in T1/T2 and N0 $(P<0.001$ and $P<0.001$, respectively) (Table 1$)$. In addition, ALDH1A1 was significantly correlated with MMP-9 in 216 gastric carcinoma specimens. Of 108 patients with low ALDH1A1 expression, 81 patients (75.0\%) had low MMP-9 expression, while 71 of 108 patients (65.7\%) with high ALDH1A1 expression also had high MMP-9 expression $(P<0.001)$ (Figure 3$)$.

We further explored the influence of tumor invasiveness on the prognostic value of ALDH1A1 expression in gastric cancer by using MMP-9 as an indicator for the invasive potential of individual tumor cells. All the patients were stratified into either a low invasiveness subgroup (low MMP-9 expression; $\mathrm{n}=118$ ) or a high invasiveness subgroup (high MMP-9 expression; $\mathrm{n}=98$ ) according to the MMP-9 expression index. Kaplan-Meier survival curves were then plotted to investigate the association between ALDH1A1 status and survival (Figure 4). In the low invasiveness subgroup, ALDH1A1 overexpression was associated with shorter OS $(P<0.001)$ and RFS $(P<0.001)$ compared with the OS and RFS in patients with low ALDH1A1 expression (Figure 4A). In the high tumor invasiveness subgroup (Figure 4B), patients with ALDH1A1 overexpression were prone to death $(P<0.001)$ and relapse $(P<0.001)$. Furthermore, the 5-year survival rate was significantly lower in the low invasiveness subgroup with ALDH1A1 overexpression (23.8\%) than that in the high invasiveness subgroup with low ALDH1A1 expression (53.0\%; $P=$ 0.002; data not shown). Therefore, the expression of ALDH1A1 appears to be a strong postoperative prognostic parameter for patients with gastric cancer independent of tumor invasiveness.

\section{Discussion}

In the present study, the expression of ALDH1A1 was investigated in 216 gastric carcinoma tissues by immunohistochemistry. We found that ALDH1A1 was significantly associated with depth invasion, lymph node metastasis and stage of disease. In addition, the KaplanMeier survival analysis revealed that the survival times (OS and RFS) of gastric cancer patients with high ALDH1A1 expression were significantly shorter than those with low ALDH1A1 expression. The prognostic value of ALDH1A1 in different subgroups according to tumor size, depth of invasion and lymph node metastasis was also estimated, which appears that ALDH1A1 may serve as a powerful prognostic factor for patients with gastric cancer in different risk groups. Furthermore, the multivariate Cox model analysis indicated that ALDH1A1 status was an independent factor for both prognosis indexes (OS and RFS) in gastric cancer. This finding suggests that ALDH1A1 plays an important role in tumor prognosis, concludes ALDH1A1 could be a potential prognostic factor of gastric cancer. Our results were consistent with previously reported results. In several investigations, it has been shown that the abnormal expression of ALDH1A1 in cancer cells is associated with tumor progression. Wakamatsu et al. [19] revealed that ALDH1 was overexpression and had positively correlated with depth invasion and TNM stage in gastric cancer, moreover, ALDH1 positivity was significantly higher in diffuse-type lymph node metastasis than that in the primary tumor. CharafeJauffret et al. [20] reported that the ALDH1A1-positive 


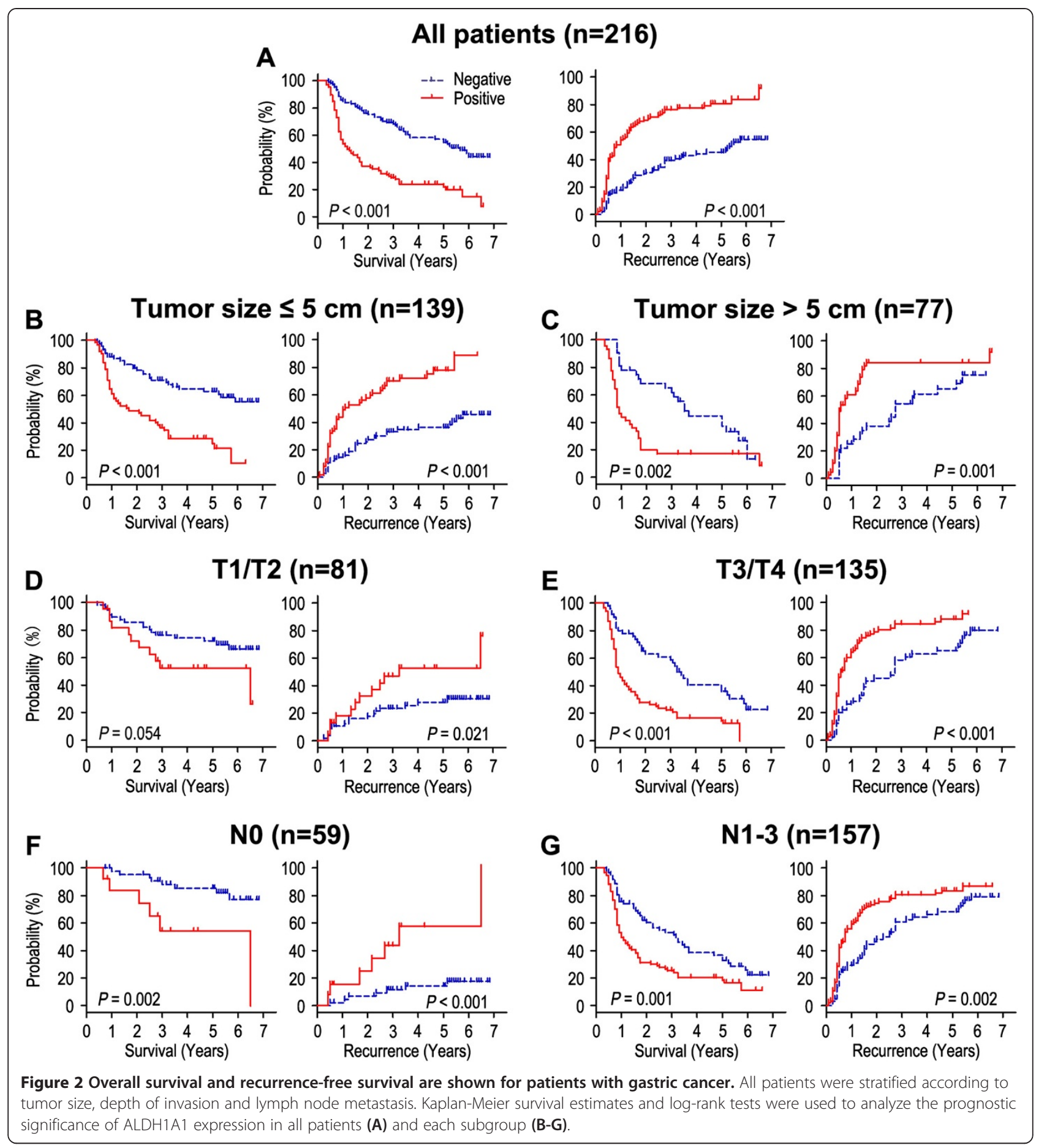

breast cancer cells were able to promote tumor invasion in vitro and tumor metastasis in mouse xenografts, moreover, expression of ALDH1A1 was an independent predictive factor for early metastasis and decreased survival in inflammatory breast cancer. Jiang et al. [14] showed that the ALDH1A1-positive lung cancer cells could generate tumors in vivo, furthermore, the expression of ALDH1A1 was positively correlated with the stage and grade of lung tumors and related to a poor prognosis for the patients with early-stage lung cancer, which suggested that ALDH1A1 could be a potential prognostic factor and therapeutic target for treatment of the patients with lung cancer. However, Dimou et al. [21] found the contradictory results that the ALDH1A1-negative expression of lung cancer patients had shorter survival compared with those with ALDH1A1-positive expression, which indicated that 
A

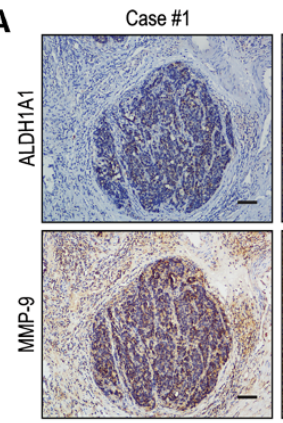

Case \#2

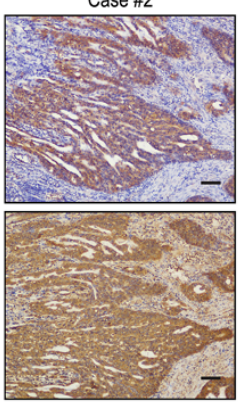

B

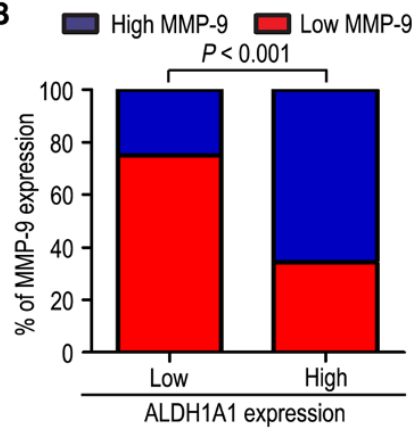

Figure 3 ALDH1A1 and MMP-9 levels correlated in $\mathbf{2 1 6}$ gastric cancer tissues. (A, B) IHC staining for ALDH1A1 and MMP-9 was performed in tumors from 216 gastric cancer patients. Representative examples of ALDH1A1 and MMP-9 staining in serial sections from the same tumor samples are shown in (A), and percentages of samples displaying low or high ALDH1A1 expression relative to MMP-9 level is shown in (B). The scale bar represents $200 \mu \mathrm{m}$.

ALDH1A1 overexpression was associated with favorable outcome.

It has been known that degradation of extracellular matrix (ECM) was a signal for the beginning of invasion and metastasis, and MMPs are important molecules involved in ECM degradation during invasion and metastasis [22]. Chu et al. [16] reported that cancer MMP9 was significantly correlated with depth of invasion and lymph node metastasis and MMP-9-positive gastric cancer patients had worse outcomes than those with MMP-9negative tumors. Zhao et al. [23] found that MMP-9

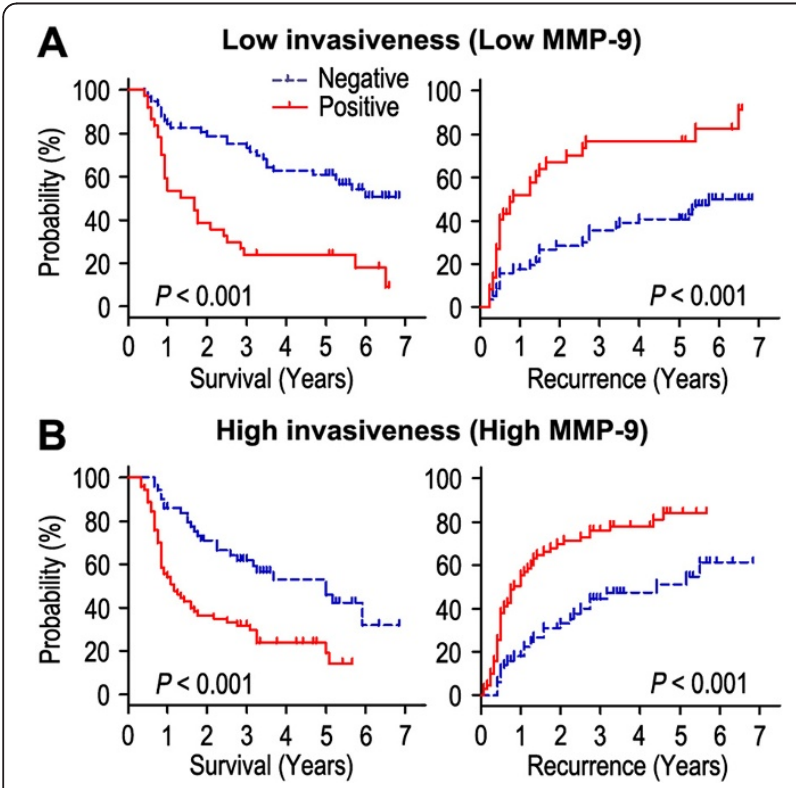

Figure 4 Overall survival and recurrence-free survival are shown for patients with low tumor invasiveness (A) and high tumor invasiveness (B). Kaplan-Meier survival estimates and log-rank tests were used to analyze the association between ALDH1A1 expression and overall survival or recurrence-free survival in patients with low invasiveness (low MMP-9; $\mathrm{n}=118$ ) or high invasiveness (high MMP-9; $n=98$ ). targeted RNA interference was able to successfully suppress MMP-9 expression and inhibit cell growth and invasion of SGC7901 gastric cancer in vitro and in vivo. Our results demonstrated that the expression of ALDH1A1 and MMP-9 was correlated with each other, indicating higher invasive and metastasizing activity in ALDH1A1 overexpression cancer cells. In addition, ALDH1A1 was highly expressed in depth of invasion, especially in T3 and T4 carcinomas, which was consistent with previously reported results [19]. As far as lymph node status was concerned, the patients with lymph node metastasis tend to show elevated ALDH1A1 expression. Collectively, ALDH1A1 status in gastric cancer promoting tumor aggressiveness suggests that ALDH1A1 could be a feasible target in cancer therapy.

\section{Conclusions}

In this study, we demonstrated that ALDH1A1 may play an important role in tumor invasion, metastasis and prognosis, and could work as a promising target for prognostic prediction in gastric cancer. Determination of ALDH1A1 expression may help to identify high-risk gastric cancer patients and thus aid the selection of appropriate therapies. Further investigation is necessary to clarify the role of ALDH1A1 in the development of gastric cancer.

\section{Competing interests}

The authors declare that they have no competing interests.

\section{Authors' contributions}

XSL, WSL, QX participated in the study conception, design, case selection and experiments. XSL, WSL and XYF carried out the data collection. QX, XYF and XSL performed the scoring of immunohistochemical staining. XSL and WSL performed the data analysis and writing of the manuscript. All the authors read and approved the final manuscript.

\section{Acknowledgements}

We gratefully acknowledge the clinical data provided by the pathology department (Affiliated Hospital of Guilin Medical University). 


\section{Author details}

${ }^{1}$ Department of Gastroenterology, Affiliated Hospital of Guilin Medical University, Guilin 541004, Guangxi Zhuang Autonomous Region, China. ${ }^{2}$ Guilin Medical University, Guilin 541004, Guangxi Zhuang Autonomous Region, China. ${ }^{3}$ Department of Spleen and stomach diseases, The First Affiliated Hospital of Guangxi University of Chinese Medicine, Nanning 530200, Guangxi Zhuang Autonomous Region, China.

Received: 20 June 2014 Accepted: 22 September 2014

Published: 24 September 2014

\section{References}

1. Compare D, Rocco A, Nardone G: Risk factors in gastric cancer. Eur Rev Med Pharmacol Sci 2010, 14:302-308.

2. Dikken JL, van Sandick JW, Maurits Swellengrebel HA, Lind PA, Putter H, Jansen EP, Boot H, van Grieken NC, van de Velde CJ, Verheij M, Cats A: Neo-adjuvant chemotherapy followed by surgery and chemotherapy or by surgery and chemoradiotherapy for patients with resectable gastric cancer (CRITICS). BMC Cancer 2011, 11:329.

3. Cunningham D, Allum WH, Stenning SP, Thompson JN, Van de Velde CJ, Nicolson M, Scarffe JH, Lofts FJ, Falk SJ, Iveson TJ, Smith DB, Langley RE, Verma M, Weeden S, Chua YJ, Participants MT: Perioperative chemotherapy versus surgery alone for resectable gastroesophageal cancer. N Engl J Med 2006, 355:11-20.

4. Nobili S, Bruno L, Landini I, Napoli C, Bechi P, Tonelli F, Rubio CA, Mini E, Nesi G: Genomic and genetic alterations influence the progression of gastric cancer. World J Gastroenterol 2011, 17:290-299.

5. Yasui W, Sentani K, Sakamoto N, Anami K, Naito Y, Oue N: Molecular pathology of gastric cancer: research and practice. Pathol Res Pract 2011 207:608-612.

6. Bornschein J, Rokkas T, Selgrad M, Malfertheiner P: Gastric cancer: clinical aspects, epidemiology and molecular background. Helicobacter 2011, 16(Suppl 1):45-52

7. Marchitti SA, Brocker C, Stagos D, Vasiliou V: Non-P450 aldehyde oxidizing enzymes: the aldehyde dehydrogenase superfamily. Expert Opin Drug Metab Toxicol 2008, 4:697-720.

8. Vasiliou V, Nebert DW: Analysis and update of the human aldehyde dehydrogenase (ALDH) gene family. Hum Genomics 2005, 2:138-143.

9. Black WJ, Stagos D, Marchitti SA, Nebert DW, Tipton KF, Bairoch A, Vasiliou V: Human aldehyde dehydrogenase genes: alternatively spliced transcriptional variants and their suggested nomenclature. Pharmacogenet Genomics 2009, 19:893-902.

10. Douville J, Beaulieu R, Balicki D: ALDH1 as a functional marker of cancer stem and progenitor cells. Stem Cells Dev 2009, 18:17-25.

11. Muzio G, Maggiora M, Paiuzzi E, Oraldi M, Canuto RA: Aldehyde dehydrogenases and cell proliferation. Free Radic Biol Med 2012, 52:735-746.

12. Ginestier $C$, Hur MH, Charafe-Jauffret $E$, Monville F, Dutcher J, Brown M, Jacquemier J, Viens P, Kleer CG, Liu S, Schott A, Hayes D, Birnbaum D, Wicha MS, Dontu G: ALDH1 is a marker of normal and malignant human mammary stem cells and a predictor of poor clinical outcome. Cell Stem Cell 2007, 1:555-567.

13. Deng S, Yang X, Lassus H, Liang S, Kaur S, Ye Q, Li C, Wang LP, Roby KF, Orsulic S, Connolly DC, Zhang Y, Montone K, Butzow R, Coukos G, Zhang L: Distinct expression levels and patterns of stem cell marker, aldehyde dehydrogenase isoform 1 (ALDH1), in human epithelial cancers. PLoS One 2010, 5:e10277.

14. Jiang F, Qiu Q, Khanna A, Todd NW, Deepak J, Xing L, Wang H, Liu Z, Su Y, Stass SA, Katz RL: Aldehyde dehydrogenase 1 is a tumor stem cell-associated marker in lung cancer. Mol Cancer Res 2009, 7:330-338.

15. Wang Y, Zhe H, Gao P, Zhang N, Li G, Qin J: Cancer stem cell marker ALDH1 expression is associated with lymph node metastasis and poor survival in esophageal squamous cell carcinoma: a study from high incidence area of northern China. Dis Esophagus 2012, 25:560-565.

16. Chu D, Zhang Z, Li Y, Zheng J, Dong G, Wang W, Ji G: Matrix metalloproteinase- 9 is associated with disease-free survival and overall survival in patients with gastric cancer. Int J Cancer 2011, 129:887-895.

17. Edge SB, Compton CC: The American Joint Committee on Cancer: the 7th edition of the AJCC cancer staging manual and the future of TNM. Ann Surg Oncol 2010, 17:1471-1474.
18. Wang J, Cui S, Zhang X, Wu Y, Tang H: High expression of heat shock protein 90 is associated with tumor aggressiveness and poor prognosis in patients with advanced gastric cancer. PLOS One 2013, 8:e62876.

19. Wakamatsu Y, Sakamoto N, Oo HZ, Naito Y, Uraoka N, Anami K, Sentani K, Oue N, Yasui W: Expression of cancer stem cell markers ALDH1, CD44 and CD133 in primary tumor and lymph node metastasis of gastric cancer. Pathol Int 2012, 62:112-119.

20. Charafe-Jauffret E, Ginestier C, lovino F, Tarpin C, Diebel M, Esterni B, Houvenaeghel G, Extra JM, Bertucci F, Jacquemier J, Xerri L, Dontu G, Stassi G, Xiao Y, Barsky SH, Birnbaum D, Viens P, Wicha MS: Aldehyde dehydrogenase 1-positive cancer stem cells mediate metastasis and poor clinical outcome in inflammatory breast cancer. Clin Cancer Res 2010, 16:45-55.

21. Dimou A, Neumeister $V$, Agarwal S, Anagnostou V, Syrigos K, Rimm DL: Measurement of aldehyde dehydrogenase 1 expression defines a group with better prognosis in patients with non-small cell lung cancer. Am J Pathol 2012, 181:1436-1442.

22. Nelson AR, Fingleton B, Rothenberg ML, Matrisian LM: Matrix metalloproteinases: biologic activity and clinical implications. J Clin Oncol 2000, 18:1135-1149.

23. Zhao F, Zhang Q, Kang C, Cui X, Wang T, Xu P, Zhou X, Liu J, Song X: Suppression of matrix metalloproteinase- 9 expression by RNA interference inhibits SGC7901 gastric adenocarcinoma cell growth and invasion in vitro and in vivo. Med Oncol 2010, 27:774-784.

doi:10.1186/1471-2407-14-705

Cite this article as: Li et al:: ALDH1A1 overexpression is associated with the progression and prognosis in gastric cancer. BMC Cancer 2014 14:705.

\section{Submit your next manuscript to BioMed Central and take full advantage of:}

- Convenient online submission

- Thorough peer review

- No space constraints or color figure charges

- Immediate publication on acceptance

- Inclusion in PubMed, CAS, Scopus and Google Scholar

- Research which is freely available for redistribution 